\title{
Determinants of outcomes in chronic pediatric peritoneal dialysis: a single center experience
}

\author{
Tariq Asi ${ }^{1 \oplus}$, Ali Düzova ${ }^{2 \oplus}$, Hasan S. Doğan ${ }^{1 \oplus}$, Gökhan Karakurt $^{1 \oplus}$,

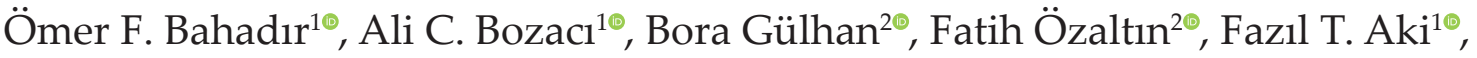 \\ Serdar Tekgül ${ }^{1 \oplus}$, Rezzan Topaloğlu ${ }^{2 \odot}$ \\ Deparments of ${ }^{1}$ Urology and ${ }^{2}$ Pediatric Nephrology, Hacettepe University Faculty of Medicine, Ankara, Turkey.
}

\begin{abstract}
Background. In situations where it may take a long time to perform renal transplantation peritoneal dialysis may become a long-term maintenance treatment, especially in countries with low donor rates. Therefore, we aimed to evaluate peritonitis, catheter revision and survival rates in children on chronic peritoneal dialysis (CPD); and to define related factors in a single tertiary center from a WHO upper middle income country.

Methods. Between January 1998 and September 2018, data of pediatric patients receiving CPD with a followup longer than 3 months were retrospectively analyzed. Demographic, clinical and catheter-related data were collected. Patients were grouped as being operated before/after 2009 in order to evaluate the effects of 2 different periods on outcomes.
\end{abstract}

Results. A total of 229 catheters in 132 patients were included in the study. The female to male ratio was 60/72. The mean age at the time of dialysis was $8.9 \pm 5.5$ years. The median follow-up period was 22.5 months (IQR 8.25-50; range 3-139).

Peritonitis incidence in 1998-2008 and 2009-2018 periods was 0.13 episodes/patient-year and 0.09 episodes/ patient-year, respectively. The overall revision rate was 1 per 46.7 patient-months. Peritonitis history was the only independent risk factor for access revision $(\mathrm{p}=0.003)$.

Peritoneal dialysis failure was observed in 25\% (33/132) of patients. The need for catheter revision due to any cause, the presence of peritonitis, history of HD and infancy were independent risk factors for PD failure. The overall mortality rate was $15.2 \%(20 / 132)$. Having a history of temporary PD catheter placement and being infant were independent risk factors for mortality.

Conclusions. Access revision is still an important complication leading to PD failure despite the development of surgical techniques. Peritonitis is the most important cause of access revision and PD failure.

Key words: end stage renal disease, peritoneal dialysis, children, complication, outcome.

Incidence of end-stage renal disease (ESRD) and the need for renal replacement therapy (RRT) in children differs worldwide due to differences in etiology of kidney disease and the financial situation to treat affected children. ${ }^{1,2}$ The incidence of ESRD in patients younger than 19 years worldwide was reported to be 4 to 18 per million of the age-related population (pmarp),

\section{Tariq Asi \\ tarikasi07@gmail.com}

Received 5th February 2020, accepted 7th May 2020. with the prevalence of RRT ranging from 18 to 100 pmarp. ${ }^{3}$ The prevalence and incidence of ESRD in Turkey in all ages were 870 and 138 per million population, respectively. ${ }^{4}$ The CREDIT-C study which is a population-based survey demonstrated that the prevalence of chronic kidney disease (CKD) stages 3-5 in children aged 5-18 was 2,600 (95\% CI 1,100$5,100)$ pmarp. ${ }^{5}$

Currently, the best choice for RRT in children is transplantation due to better survival 
and quality of life. ${ }^{3}$ When this choice is not possible chronic peritoneal dialysis (CPD) or hemodialysis (HD) could be the only feasible option as a bridge therapy until transplantation with no difference in morbidity or mortality being reported between them. ${ }^{6} \mathrm{CPD}$ conserves the vascular pool required for long-term RRT and allows more individual liberty compared to HD. However, the practicability of this choice highly depends on the families' readiness and accommodation to take liability for ambulatory medical care. Also, despite the broad admissibility of CPD as the RRT modality of choice for children, it is impeded to a great degree by several complications like catheter revisions, infections and ultrafiltration insufficiency which may lead to dialysis failure.

With low organ donors rate in some countries, dialysis has become a final treatment destination rather than being a bridge to transplantation. More than one third of the patients on CPD are expected to shift to HD within 2 years and less than $40 \%$ of them are anticipated to continue beyond this point. ${ }^{7}$ In this study, we aimed to evaluate peritonitis, catheter revision and survival rates; and to define related factors among pediatric patients on CPD in a single tertiary center from a WHO upper middle income country over the last 20 years.

\section{Material and Methods}

After obtaining ethical committee approval (GO19/1063)-Hacettepe University, we retrospectively analyzed the departmental records of pediatric patients with CPD in our center between 1998 and 2018. Patients equal to or younger than 20 years of age at the start of dialysis with a follow-up longer than 3 months were included in the study. We collected demographic data [age, gender, operation period (before or after 2009), etiology of ESRD, age at dialysis time and at last follow-up], clinical data including weight, height, BMI, presence of anorectal malformations, constipation, dialysis history before PD catheter replacement, time to first catheter use (break-in period), CPD program, exit-site or tunnel infection (ESI/ TI) history, peritonitis attacks and culture results, time to first and second access revision and their causes, time to transplantation, PD failure and patient survival rates; and catheter related data [intra-abdominal (felt vs. curled) and extra-peritoneal (straight vs. swan-neck) configurations].

To better understand whether 2 different periods have effects on access revision, peritonitis or mortality rates, we labeled the patients being operated before/after 2009, the year which represents a midpoint of years and patients. BMI standard deviation scores (SDS) were calculated using CDC charts and according to the results patients were classified as underweight, normal or overweight. Depending on the International Society for Peritoneal Dialysis (ISPD) guidelines we put break-in periods as less or more than 14 days. Peritonitis was defined according to the recommendations of the ISPD. ${ }^{8}$ Mechanical dysfunction was defined as catheter dysfunction caused by catheter migration, omental obstruction or occlusion by fibrin clots. Access revision occurs within 4 weeks of previous revision was considered as recurrent. PD failure is shifting to HD because of peritonitis, insufficient dialysis or mechanical dysfunction.

All patients received antibiotics perioperatively at the time of induction of general anesthesia. Most of the time a first generation cephalosporin or an amino-penicillin was the choice. Postoperatively $10 \mathrm{ml} / \mathrm{kg}$ of a dilute solution of heparin and dialysate $(0.5 \mathrm{unit} / 1 \mathrm{ml})$ was used to wash the catheter at the first 0-, 16and 24-hours to prevent clot formation.

All statistical analyses were performed using Statistical Package for Social Sciences (SPSS) 22.0 for windows. Student's t test and Mann-Whitney $\mathrm{U}$ test were used to compare non-categorical variables; Chi-square test and Fisher's exact test were used to compare categorical variables. We used logistic regression modeling to find predictors for access revision. Survival analyzed using Kaplan-Meier curves. To define 
factors predicting PD failure and mortality, Cox proportional modeling was used. Minimal statistical significance was defined as $p$ values $<0.05$.

\section{Results}

A total of 229 catheters in 132 patients were included in the study. Demographics and clinical characteristics are shown in Table I. There were 60 females and 72 males, with a mean age of $6.0 \pm 4.9$ years (range $1-240$ months) at time of ESRD diagnosis and $8.9 \pm 5.5$ years at time of dialysis. 16 patients were infants ( $<1$ year of age) with an average age of $5.5 \pm 4.9$ months. The median follow-up period was 22.5 months (IQR 8.25-50; range 3-139); $18.2 \%$ and $5.3 \%$ of patients were underweight and overweight, respectively. Rates of being underweight was not statistically different between infants and older age group $(33.7 \%$ vs. $17.1 \%, p=0.161)$. Congenital anomalies of kidney and urinary tract (CAKUT) were the etiology of ESRD in 59 patients $(44.7 \%)$. Thirty-seven $(28.0 \%)$ of patients had emergent need to dialysis due to electrolyte abnormalities, acidosis or edema; $64.9 \%$ (24/37) of these patients needed HD while the rest had a temporary PD catheter placed in intensive care unit conditions. Additional 13 patients were electively started on HD and transitioned to PD thereafter, resulting in $28.0 \%$ (37/132) of patients with history of HD. Constipation was documented in 13 (9.8\%) patients.

Catheters with curled end and swan-neck configuration were the choice in 72 (54.5\%) patients. The average break-in period was 16 days (IQR 8-20; range 1-150 days). PD program was known in 123 patients; all patients started with continuous ambulatory PD (CAPD) program and 28 patients shifted to automated PD (APD) program thereafter.

\section{Peritonitis}

Seventy-three $(55.3 \% ; 73 / 132)$ of patients had at least one attack of peritonitis with a 142 attacks at total, making the mean attack number of all patients as $1.07 \pm 1.47$ episodes per patient.
Table I. Demographic and clinical patient characteristics.

\begin{tabular}{ll}
\hline Characteristic & Results \\
Gender, $\mathrm{n}(\%)$ & \\
\hline Female & $60(45.4)$ \\
Male & $72(54.6)$ \\
Age at ESRD diagnosis, years & $6.0 \pm 4.9$ \\
Age at the start of dialysis, years & $8.9 \pm 5.5$ \\
Patients $<1$ year of age, $\mathrm{n}(\%)$ & $16(12.1)$ \\
Median follow-up period, months & $22.5(8.25-50.0)$ \\
Height, cm & $116 \pm 32 \mathrm{~cm}$ \\
BMI SD score & \\
Underweight & $24(18.2)$ \\
Normal & $95(72.0)$ \\
Overweight & $7(5.3)$ \\
Unknown & $6(4.5)$ \\
Operation period, n (\%) & $67(50.8)$ \\
Before 2009 & $65(49.2)$ \\
After 2009 & \\
Diagnosis, $\mathrm{n}(\%)$ & $59(44.7)$ \\
CAKUT & $20(15)$ \\
Obstructive & $14(10.6)$ \\
Refluxive & $6(4.5)$ \\
Neurogenic & $5(3.8)$ \\
Renal hypo-/dysplasia & $14(10.6)$ \\
Cystic renal disease & $73(55.3)$ \\
Non-CAKUT & $47(35.6)$ \\
Glomerulopathy & $18(13.6)$ \\
Metabolic & $8(6.1)$ \\
Miscellaneous & $37(28.0)$ \\
History of HD, $\mathrm{n}$ (\%) & $14(10.6)$ \\
Temporary PD catheter placement, \\
n (\%)
\end{tabular}

ESRD: end-stage renal disease, BMI SD: body mass index standard deviation, CAKUT: congenital anomalies of kidney and urinary tract, HD: hemodialysis, PD: peritoneal dialysis, CAPD: continuous ambulatory peritoneal dialysis, APD: automated peritoneal dialysis. 
Peritonitis incidence in 1998-2008 period was 0.13 episode/patient-year and decreased to 0.09 episode/patient-year in 2009-2018 period. Sixtynine $(69 / 142 ; 48.6 \%)$ and $22(22 / 142 ; 15.5 \%)$ of peritoneal fluid cultures had gram positive and gram negative bacteria, respectively. The rest $(35.9 \%)$ had negative culture results. Staphylococcus consisted 78.3\% (54/69) of gram positive culprits while Pseudomonas consisted $31.8 \%$ (7/22) gram negative ones. 23.3\% (17/73) of patients with peritonitis underwent access revision whether by removing the catheter $(4 / 17)$ or replacing a new one in the same session (13/17). Among 73 patients with peritonitis, 39.7\% (29/73) had access revision at some point of their follow-up due to mechanical dysfunction. Having ESI/TI was the only significant predictor for peritonitis at some point of follow-up (73.9\% vs. $51.4 \% \mathrm{p}=0.048)$. Additionally, there was a direction heading towards significance between peritonitis and APD program and being operated before 2009 (Table II).

ESI/TIs were noticed in $17.4 \%$ (23/132) of patients and treated with oral antibiotics. Patients on APD program had higher exit-site or tunnel infection (ESI/TI) rate [35.7\% (10/28) vs. $13.7 \%$ (13/95), $p=0.009]$. Interestingly most of these patients had catheters with straight extraperitoneal orientation compared to patients on CAPD program who had more swan-neck catheters $[66.7 \%(12 / 18)$ vs. $26.2 \% \quad(22 / 84)$, $\mathrm{p}=0.002]$. Additionally, being operated before 2009 was another significant risk factor for ESI/ TI [28.4\% (19/67) vs. 6.2\% (4/65), $\mathrm{p}=0.001]$.

\section{Revision Rate}

Access revision rate was 51.5\% (68/132). Second and third revision were needed in $36.8 \%(25 / 68)$ and $5.9 \%(4 / 68)$ of these patients, respectively, resulting in an average revision rate of 1 per 46.7 patient-months. While $75 \%(51 / 68)$ of first and $68 \%(17 / 25)$ of second revisions were due to mechanical failure, peritonitis was the reason in $25 \%(17 / 68)$ and $32 \%(8 / 25)$ of them, respectively. The rest were due to extravasations. Average time to first and second revisions was $9.7 \pm 18.1$ and $11.9 \pm 18$ months, respectively. $67.6 \%(46 / 68)$ had early revision (within 90 days). $28.0 \%$ (7/25) of second revisions were recurrent and all of these recurrent revisions occurred after revision due to mechanical failure. Demographic and clinical differences between patients with or without access revision are given in Table II. Including ESRD etiology, constipation and peritonitis in logistic regression analysis, the only independent predictor for access revision was peritonitis history (OR 2.8; 95\% CI 1.3 to 5.9; $\mathrm{p}=0.003$ ).

\section{PD Failure}

During the observation period, $25.0 \%$ (33/132) of patients permanently shifted to HD after a median of 24 months (IQR 6.5-54); while $45.5 \%$ $(15 / 33)$ of them shifted after peritonitis, $45.5 \%$ $(15 / 33)$ of them shifted after mechanical failure. Inadequate dialysis was the reason in the other $3(9.0 \%)$ patients. When we compared patients with PD failure (33/132) with patients who are still on PD (24/132), PD failure was significantly higher in patients operated before 2009 [80.8\% $(21 / 26)$ vs. $38.7 \%$ (12/31), $\mathrm{p}=0.001$ ] despite there was no significant difference in peritonitis or revision rates between patients operated before or after 2009. The need for catheter revision due to any cause (HR 3.65; 95\% CI 1.19 to 11.17 ; $\mathrm{p}=0.023$ ), the presence of peritonitis (HR 3.46; $95 \%$ CI 1.11 to $10.78, p=0.032$ ), history of HD (HR 2.58; 95\% CI 1.09 to $6.13, \mathrm{p}=0.032$ ) and infancy (HR 5.2; 95\% CI 1.02 to 26.70, $\mathrm{p}=0.047$ ) were independent risk factors for PD failure on multivariate analysis (Table III). There was no significant difference in PD failure between early or late revisions. PD technique survival rate at $1-, 5$ - and 10 - year period was $75 \%, 35 \%$ and $10 \%$, respectively (Fig. 1).

Forty-six (46/132, 34.8\%) patients on PD had transplantation after a median of 26 months (IQR 15.75-66.25 months), and PD catheter was removed after an average of $6 \pm 3$ weeks. Out of 33 patients who shifted to HD 10 (10/132, 7.6\%) patients had transplantation thereafter, making the median dialysis period before transplantation as 30 months (IQR 17- 
Table II. Demographic and clinical characteristics and their relation to access revision and peritonitis.

\begin{tabular}{|c|c|c|c|c|c|}
\hline Parameter & & $\begin{array}{l}\text { Access revision } \\
\text { rate }\end{array}$ & $p$ & Peritonitis rate & $p$ \\
\hline \multirow[t]{2}{*}{ Gender } & Male (72) & $47.2 \%(34 / 72)$ & 0.280 & $54.2 \%(39 / 72)$ & 0.774 \\
\hline & Female (60) & $56.7 \%(34 / 60)$ & & $56.7 \%(34 / 60)$ & \\
\hline \multirow[t]{2}{*}{ Date } & $<2009(67)$ & $52.2 \%(35 / 67)$ & 0.866 & $62.7 \%(42 / 67)$ & 0.083 \\
\hline & $>2009(65)$ & $50.8 \%(33 / 65)$ & & $47.7 \%(31 / 65)$ & \\
\hline \multirow[t]{2}{*}{ Age } & Infant (16) & $56.3 \%(9 / 16)$ & 0.686 & $56.3 \%(9 / 16)$ & 0.935 \\
\hline & Others (116) & $50.9 \%(59 / 119)$ & & $55.2 \%(64 / 116)$ & \\
\hline \multirow[t]{2}{*}{ ESRD etiology } & CAKUT (59) & $59.3 \%(35 / 59)$ & 0.107 & $59.3 \%(35 / 59)$ & 0.404 \\
\hline & Non-CAKUT (73) & $45.2 \%(33 / 73)$ & & $52.1 \%(38 / 73)$ & \\
\hline \multirow[t]{2}{*}{ Constipation } & Yes (13) & $76.9 \%(10 / 13)$ & 0.078 & $61.5 \%(8 / 13)$ & 0.772 \\
\hline & No (119) & $48.7 \%(58 / 119)$ & & $54.6 \%(65 / 119)$ & \\
\hline \multirow[t]{2}{*}{ BMI } & Underweight (24) & $62.5 \%(15 / 24)$ & 0.234 & $50 \%(12 / 24)$ & 0.602 \\
\hline & Others (102) & $49 \%(50 / 102)$ & & $55.9 \%(57 / 102)$ & \\
\hline \multirow[t]{2}{*}{ History of HD } & Yes (40) & $56.8 \%$ & 0.452 & $48.6 \%$ & 0.337 \\
\hline & No (92) & $49.5 \%$ & & $57.9 \%$ & \\
\hline \multirow{2}{*}{$\begin{array}{l}\text { Temporary PD catheter } \\
\text { placement }\end{array}$} & Yes (14) & $35.7 \%(5 / 14)$ & 0.264 & $57.1 \%(8 / 14)$ & 0.884 \\
\hline & No (118) & $53.4 \%(63 / 118)$ & & $55.1 \%(65 / 118)$ & \\
\hline \multirow[t]{2}{*}{ Break-in period (days) } & $>14(57)$ & $47.4 \%(27 / 57)$ & 0.645 & $56.1 \%(32 / 57)$ & 0.499 \\
\hline & $<14(64)$ & $51.6 \%(33 / 64)$ & & $50 \%(32 / 64)$ & \\
\hline \multirow{2}{*}{$\begin{array}{l}\text { Catheter configuration } \\
\text { (Curled Swan-neck) }\end{array}$} & Yes (72) & $52.8 \%(38 / 72)$ & 0.493 & $51.4 \%(37 / 72)$ & 0.784 \\
\hline & No (35) & $45.7 \%(16 / 35)$ & & $48.6 \%(17 / 35)$ & \\
\hline \multirow[t]{2}{*}{ PD program } & CAPD (95) & $54.7 \%(52 / 95)$ & 0.296 & $52.6 \%(50 / 95)$ & 0.154 \\
\hline & $\mathrm{APD}(28)$ & $42.9 \%(12 / 28)$ & & $67.9 \%(19 / 28)$ & \\
\hline \multirow[t]{2}{*}{$\mathrm{ESI} / \mathrm{TI}$} & Yes (23) & $52.2 \%(12 / 23)$ & 0.945 & $73.9 \%(17 / 23)$ & 0.048 \\
\hline & No (109) & $51.4 \%(56 / 109)$ & & $51.4 \%(56 / 109)$ & \\
\hline \multirow[t]{2}{*}{ Peritonitis } & Yes (73) & $63 \%(46 / 73)$ & 0.003 & & \\
\hline & No (59) & $37.2 \%(22 / 59)$ & & & \\
\hline
\end{tabular}

ESRD: end-stage renal disease, CAKUT: congenital anomalies of kidney and urinary tract, BMI: body mass index, HD: hemodialysis, PD: peritoneal dialysis, CAPD: continuous ambulatory peritoneal dialysis, APD: automated peritoneal dialysis, ESI/TI: exit-site infection/tunnel infection.

Table III. Cox proportional hazard modeling of factors affecting peritoneal dialysis failure.

\begin{tabular}{lcc}
\hline Variable & Hazard ratio (95\% confidence interval) & $P$ \\
\hline Operation period (before 2009) & $1.56(0.69-3.49)$ & 0.282 \\
History of hemodialysis & $2.58(1.09-6.13)$ & 0.032 \\
Age $<1$ year & $5.2(1.02-26.70)$ & 0.047 \\
Presence of peritonitis & $3.46(1.11-10.78)$ & 0.032 \\
Presence of revision & $3.65(1.19-11.17)$ & 0.023 \\
\hline
\end{tabular}

66.5 months); $18.2 \%(24 / 132)$ of patients still having PD with a median of 15 months (IQR $8-50.75$ months); $6.9 \%$ (9/132) of patients lost to follow up. The median dialysis period before transplantation shortened with time [before 2009 median 37 months (IQR13.25-74.5) while after 2009 median 26 months (IQR 18-54.5), p= $0.3]$. 


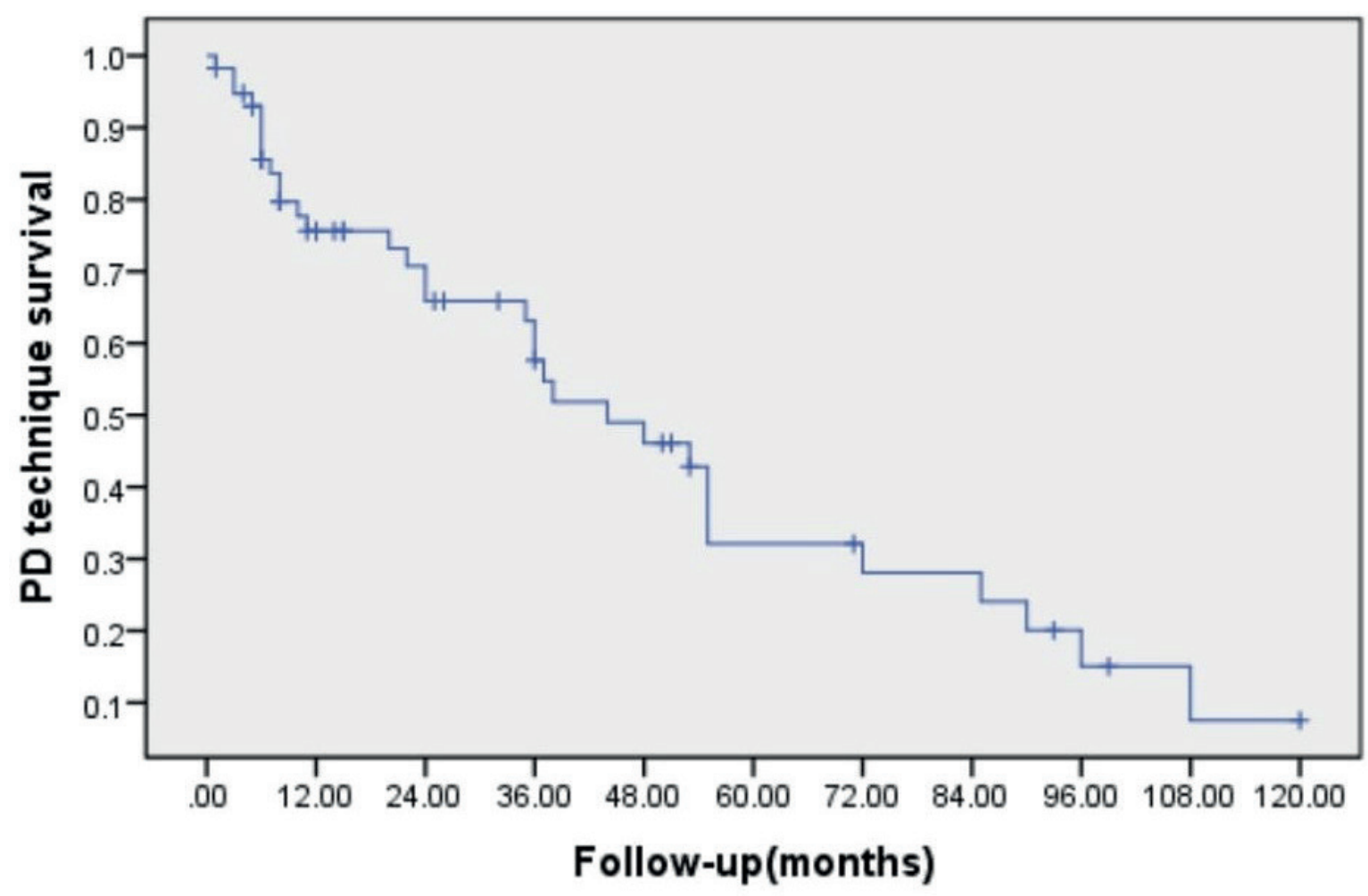

PD technique survival rate decreases with follow-up

Fig. 1. PD technique survival rate during follow-up period.

\section{Mortality}

Twenty (20/132; 15.2\%) patients died after a median PD period of 13.5 months (IQR 2.5-43.5); seven of them were infants. Thirty percent $(6 / 20)$ of them were dialysis-related (four peritonitis, one hypertensive encephalopathy, one cardiac tamponade). Pneumonia was the cause in two patients. Data related to the etiology in the rest was not available. In multivariate analysis, having a history of temporary PD catheter placement (HR 2.98; 95\% CI 1.06 to 8.37, $\mathrm{p}=$ 0.038 ) and infancy (HR 4.05; 95\% CI, 1.58 to 10.35, $\mathrm{p}=0.003$ ) were independent risk factors for mortality. Patient survival rate at 1-, 5- and 10 -years was $92 \%, 76 \%$ and $55 \%$ respectively.

\section{Discussion}

When transplantation is not possible, peritoneal dialysis is the preferable choice in children requiring RRT due to its simplicity and utility of conserving residual renal function. But unfortunately, this modality is associated with complications like access revision, peritonitis and ultrafiltration insufficiency which may lead to technical failure. Rigoni et al. ${ }^{9}$ reported 0.4 year period for patients on PD to receive call to transplantation, this period is much longer in Turkey compared to the literature which indicates that PD has become a long-term maintenance treatment. This emphasizes the need to minimize complications and technique failure rates.

Different studies reported that ESRD etiology may have an effect on access revision, mortality and transplantation rates. ${ }^{10}$ According to TUPEPD study group's report ${ }^{11}$ related to 19892002 period, CAKUT and non-CAKUT were the etiology in $42.3 \%$ and $46.4 \%$ of Turkish children with ESRD-the rest were with unknown etiology-, with glomerulonephritis and vesicoureteral reflux disease (VUR) being the cause in 
$28.8 \%$ and $18.1 \%$ of the cases. In this study, we observed similar rates with different subgroup distribution in the CAKUT arm - lower VUR and higher obstructive anomalies-. It was postulated that these diseases may require complex urologic intervention which can lead to infectious and mechanical complications. Additionally, patients with CAKUT have better residual renal function and are less likely to develop oligo-anuria. ${ }^{12}$ In conjunction with literature data, although it did not reach to statistical significance, we noticed a tendency of patients with CAKUT to have higher revision rates $(59.3 \%$ vs. $45.2 \%, p=0.107)$ and longer time-to-transplantation period (34.5 months (IQR 16.5-75.2) vs. 28 months (IQR 17-61), $\mathrm{p}=$ 0.38 ) without any difference in mortality.

PD failure is still a major complication that precludes the continuation of peritoneal dialysis. Sampimon et al. ${ }^{13}$ studied 224 patients managed by $\mathrm{PD}$ and reported $21 \%$ rate of $\mathrm{PD}$ failure after 2 -year period. We observed a 25\% PD failure rate, with access revision and peritonitis being the major risk factors. Also, being operated before 2009 increased PD failure risk which could be related to the chronic exposure to dialysate and the peritoneal membrane injury initiated during peritonitis. A study conducted from the IPPN registry ${ }^{10}$ reported a lower access revision rate at a younger age; however, access survival in infants (<1year) and PD technique survival in patients younger than 3 months of age were poorer. Similarly, we observed a higher PD failure risk in infants $(<12$ months of age). We think that this observation could be explained by the fact that infants do not have a very well developed abdominal wall.

Access revision is considered a bothering stressful situation for surgeons to deal with, especially when the cause is mechanical. The rate of such complications is variable between different studies and ranges between 13-34\%. The major cause is also variable as these studies are related to different eras. ${ }^{10,14}$ This difference is mostly related to the adherence to better antibiotic prophylaxis guidelines, thereby lowering infection requiring revision. In our study, the only significant predictor for catheter revision was a history of peritonitis. Being a single-center experience over 20 year-period -with near one-third of our patients being operated before 2004- may explain these results. There are scarce data related to the second recurrent revision rate in pediatric $\mathrm{PD}$. The IPPN analysis ${ }^{10}$ reported $16 \%$ second recurrent revision rate, and almost all of them occurred after a revision for mechanical dysfunction. In this study, $28.0 \%$ of second revisions were recurrent. All of them occurred after a revision for mechanical dysfunction.

Peritonitis stays the main complication of PD and a primary cause for technical failure despite the reported decrease in its rates since the late $1980 \mathrm{~s} .{ }^{15}$ Its' $^{\prime}$ incidence in pediatric patients ranges between 0.21-0.71 episode/patientyear, and gram positive bacteria -especially Staphylococcus - are the culprit most of the time. ${ }^{16}$ In this study, we postulate that our lower peritonitis incidence and the decrease noticed between two periods may be related to increased adherence to early management policy and increased education in parents and patients. Additionally, as ESI/TI seems to be a pathway to develop subsequent peritonitis, any patient with ESI suspicion was started on povidone dressing, and if pus is present oral antibiotics were prescribed. Peritoneal fluid culture results are similar to the ones reported in the literature. ${ }^{15,16}$

Exit-site infection is the pathway to develop subsequent tunnel infection and peritonitis that leads to elevated rates of catheter loss, morbidity, and mortality. Swartz et al. ${ }^{17}$ reported a $17 \%$ rate of ESI for an annualized rate of 0.25 ESIs per year of PD. PD program whether plays a role in ESI or not is still debatable. ${ }^{18}$ In this study, being on an APD program besides being operated before 2009 were risk factors for ESI. Most of the patients on an APD program had PD catheter with straight extra-peritoneal orientation. 
The impact of PD catheter configuration on complication rates are still arguable. ${ }^{10,19}$ In our patients there was no significant difference in complication rates between diverse catheter configurations.

Although both the International Society for Peritoneal Dialysis (ISPD) and European guidelines recommend a break-in period of 10-15 days to prevent leaks and catheter complications, the effect of break-in period on PD outcome is still questionable. ${ }^{20}$ In this study, break-in period had no significant impact on access revision or patient survival. In our center, all patients begin with low fill volume and short dwell times which may explain these results.

Constipation in PD patients is a risk factor for peritonitis and catheter malfunction or migration. ${ }^{21}$ In this study many patients' records were deficient in data related to their bowel movements. But we noticed that patients with constipation had a strong tendency to have catheter revisions, mostly due to mechanical failure.

Its reported that the mortality of dialyzed children is declining, and mortality as a direct outcome of PD is rare. ${ }^{22}$ In the early days of RRT, younger age at the start of dialysis was associated with poor outcome. ${ }^{23}$ These results improved in recent years because of the greater attention paid to dialysis technology, nutrition and infection prevention and management. ${ }^{24}$ Body mass index (BMI) whether low or high is related to increased mortality in children with ESRD. ${ }^{25}$ We observed a $15.2 \%$ all-cause mortality rate. Being an infant and having a history of temporary PD catheter placement were associated with increased mortality. These results can be explained by the "obesity paradox" effect as $33.7 \%$ of infants were underweight ${ }^{26}$, and by the observation that most of the temporary PD catheters were placed in the intensive care unit which implies the severity of disease status.

The present study comes with limitations. Data related to omentectomy whether performed or not was not available. All patients had open procedure therefore we could not compare it with other surgical procedures. Also, as routine nasal cultures were not taken, no data were available to check the relationship between the infection rates and nasal carriage. Still, this study clearly identifies the complications of chronic PD and related risk factors in a relatively large sample size and long follow up period. Additionally, it states the expected period of $\mathrm{PD}$ before transplantation in a WHO upper middle-income country with low donor rates which will help physicians and patients in decision making process.

As a conclusion, access revision is still a bothering complication leading to PD failure despite the development of surgical techniques. Peritonitis-which is caused primarily by ESI- is the most significant reason for access revision and PD failure. Mortality is higher in patients who need temporary PD catheters. The infant age group carries higher risk in terms of both PD failure and mortality. According to these results, better strategies should be adopted to lower peritonitis rates in PD patients.

\section{REFERENCES}

1. Bertram JF, Goldstein SL, Pape L, Schaefer F, Shroff RC, Warady BA. Kidney disease in children: latest advances and remaining challenges. Nat Rev Nephrol 2016; 12: 182-191.

2. Schaefer F, Borzych-Duzalka D, Azocar M, et al; IPPN Investigators. Impact of global economic disparities on practices and outcomes of chronic peritoneal dialysis in children: insights from the International Pediatric Peritoneal Dialysis Network Registry. Perit Dial Int 2012; 32: 399-409.

3. Harambat J, van Stralen KJ, Kim JJ, Tizard EJ. Epidemiology of chronic kidney disease in children. Pediatr Nephrol 2012; 27: 363-373.

4. Seyahi N, Ates K, Süleymanlar G. Türkiye'de renal replasman tedavilerinin güncel durumu: Türk Nefroloji Derneği kayıt sistemi 2014 yılı özet raporu. Türk Nefroloji Derneği Dergisi 2016; 25: 135-141.

5. Soylemezoglu O, Duzova A, Yalcinkaya F, Arinsoy T, Suleymanlar G. Chronic renal disease in children aged 5-18 years: a population-based survey in Turkey, the CREDIT-C study. Nephrol Dial Transplant 2012; (27 Suppl 3): iii146-iii151. 
6. Wong CS, Hingorani S, Gillen DL, et al. Hypoalbuminemia and risk of death in pediatric patients with end-stage renal disease. Kidney Int 2002; 61: 630-637.

7. Chaudhary K, Sangha H, Khanna R. Peritoneal dialysis first: rationale. Clin J Am Soc Nephrol 2011; 6: 447-456.

8. Li PK, Szeto CC, Piraino B, et al. ISPD peritonitis recommendations: 2016 update on prevention and treatment. Perit Dial Int 2016; 36: 481-508.

9. Rigoni M, Torri E, Nollo G, et al. Survival and timeto-transplantation of peritoneal dialysis versus hemodialysis for end-stage renal disease patients: competing-risks regression model in a single Italian center experience. J Nephrol 2017; 30: 441-447.

10. Borzych-Duzalka D, Aki TF, Azocar M, et al; International Pediatric Peritoneal Dialysis Network (IPPN) Registry. Peritoneal dialysis access revision in children: causes, interventions, and outcomes. Clin J Am Soc Nephrol 2017; 12: 105-112.

11. Bakkaloglu SA, Ekim M, Sever L, et al. Chronic peritoneal dialysis in Turkish children: a multicenter study. Pediatr Nephrol 2005; 20: 644-651.

12. Ha IS, Yap HK, Munarriz RL, et al; International Pediatric Peritoneal Dialysis Network Registry. Risk factors for loss of residual renal function in children treated with chronic peritoneal dialysis. Kidney Int 2015; 88: 605-613.

13. Sampimon DE, Coester AM, Struijk DG, Krediet RT. The time course of peritoneal transport parameters in peritoneal dialysis patients who develop encapsulating peritoneal sclerosis. Nephrol Dial Transplant 2011; 26: 291-298.

14. Rinaldi S, Sera F, Verrina E, et al; Italian Registry of Pediatric Chronic Peritoneal Dialysis. Chronic peritoneal dialysis catheters in children: a fifteenyear experience of the Italian Registry of Pediatric Chronic Peritoneal Dialysis. Perit Dial Int 2004; 24: 481-486.

15. Chadha V, Schaefer FS, Warady BA. Dialysisassociated peritonitis in children. Pediatr Nephrol 2010; 25: 425-440.
16. Ponce D, de Moraes TP, Pecoits-Filho R, Figueiredo AE, Barretti P. Peritonitis in children on chronic peritoneal dialysis: the experience of a large National Pediatric Cohort. Blood Purif 2018; 45: 118-125.

17. Swartz SJ, Neu A, Skversky Mason A, et al. Exit site and tunnel infections in children on chronic peritoneal dialysis: findings from the Standardizing Care to Improve Outcomes in Pediatric End Stage Renal Disease (SCOPE) Collaborative. Pediatr Nephrol 2018; 33: 1029-1035.

18. Chiu MC, Tong PC, Lai WM, Lau SC. Peritonitis and exit-site infection in pediatric automated peritoneal dialysis. Perit Dial Int 2008; (28 Suppl 3): S179-S182.

19. Hagen SM, Lafranca JA, IJzermans JN, Dor FJ. A systematic review and meta-analysis of the influence of peritoneal dialysis catheter type on complication rate and catheter survival. Kidney Int 2014; 85: 920932.

20. Liu Y, Zhang L, Lin A, Ni Z, Qian J, Fang W. Impact of break-in period on the short-term outcomes of patients started on peritoneal dialysis. Perit Dial Int 2014; 34: 49-56.

21. Nikitidou O, Liakopoulos V, Kiparissi T, Divani M, Leivaditis K, Dombros N. Peritoneal dialysis-related infections recommendations: 2010 update. What is new? Int Urol Nephrol 2012; 44: 593-600.

22. Shroff R, Rees L, Trompeter R, Hutchinson C, Ledermann $S$. Long-term outcome of chronic dialysis in children. Pediatr Nephrol 2006; 21: 257-264.

23. Wood EG, Hand M, Briscoe DM, et al; North American Pediatric Renal Transplant Cooperative Study. Risk factors for mortality in infants and young children on dialysis. Am J Kidney Dis 2001; 37: 573-579.

24. Carey WA, Martz KL, Warady BA. Outcome of patients initiating chronic peritoneal dialysis during the first year of life. Pediatrics 2015; 136: e615-e622.

25. Wong CS, Gipson DS, Gillen DL, et al. Anthropometric measures and risk of death in children with end-stage renal disease. Am J Kidney Dis 2000; 36: 811-819.

26. Park J, Ahmadi SF, Streja E, et al. Obesity paradox in end-stage kidney disease patients. Prog Cardiovasc Dis 2014; 56: 415-425. 